\title{
Dipole Moment and Electronic Structure Calculations of the Electronic States of the molecular ion $\mathrm{SiN}^{+}$
}

\author{
Karam Hamdan ${ }^{1}$, Ghassan Younes $^{1} \&$ Mahmoud Korek $^{1}$ \\ ${ }^{1}$ Faculty of Science, Beirut Arab University, P. O. Box 11-5020 Riad El Solh, Beirut 1107 2809, Lebanon \\ Correspondence: Mahmoud Korek, Faculty of science, Beirut Arab University, P.O.Box 11-5020, Beirut Lebanon. \\ E-mail: mahmoud.korek@bau.edu.lb, fkorek@yahoo.com
}

Received: April 21, 2015 Accepted: May 6, 2016 Online Published: June 6, 2016

doi:10.5539/apr.v8n4p1 URL: http://dx.doi.org/10.5539/apr.v8n4p1

\begin{abstract}
A theoretical investigation of the lowest electronic states of the molecular ion $\mathrm{SiN}^{+}$has been performed via Complete Active Space Self Consistent Field (CASSCF) method with Multi Reference Configuration Interaction MRCI+Q (single and double excitations with Davidson correction) calculations. The potential energy curves of the low-lying 37 electronic states in the representation ${ }^{2 \mathrm{~s}+1} \Lambda^{(+-)}$, up to $140000 \mathrm{~cm}^{-1}$, have been investigated. The permanent dipole moment, the harmonic frequency $\omega_{\mathrm{e}}$, the equilibrium internuclear distance $\mathrm{R}_{\mathrm{e}}$, the rotational constants $B_{e}$ and the electronic energy with respect to the ground state $T_{e}$ have been calculated for these electronic states. The comparison between the values of the present work and those available in the literature for several electronic states shows a very good agreement. The permanent dipole moment, of the investigated 37 electronic states, have been calculated in the present work for the first time along with the investigation of nine new electronic states that have not been observed yet.
\end{abstract}

Keywords: $a b$ initio calculation, permanent dipole moments, electronic structure, spectroscopic constants, potential energy curves

\section{Introduction}

The remarkable interest in silicon nitride $\mathrm{SiN}$ reside in its important role in the stellar atmosphere and in many properties such as strength, hardness, chemical inertness, good resistance to corrosion, high thermal stability, and good dielectric properties (Katz, 1980). In literature many spectroscopic investigations have been focused on the ground and the first excited states where some spectroscopic constants have been obtained (Linton, 1975, Bredohl et al., 1976, Saito et al., 1983, Foster, 1984, Foster et al., 1985, Yamada \& Hirota, 1985, Yamada et al., 1988, Foster, 1989, Elhanine et al., 1992, Ito et al., 1993, Naulin et al., 1993).

In contrast, fragmented data on the molecular ion $\mathrm{SiN}^{+}$have been published in literature. By using an ab initio calculation (MRD-CI), Bruna et al. (1980) found that the ground state is $\mathrm{X}^{3} \Sigma^{-}$for this cation. By using the second-order Möller-Plesset perturbation theory, Goldberg et al. (1994) determined the energy separation $\mathrm{T}_{\mathrm{e}}$ between the ${ }^{3} \Sigma^{-}$and ${ }^{3} \Pi$ electronic states along with the internuclear distance $R_{e}$ of these electronic states for the $\mathrm{SiN}^{+}$molecular ion. From CASSCF, CMRCI, CCSDT and density functional theoretical calculations, Cai and François (1999) calculated the spectroscopic constants of the $\mathrm{SiN}^{+}$molecular ion and they found that the ${ }^{3} \Pi$ is $460 \mathrm{~cm}^{-1}$ above the ground state ${ }^{3} \Sigma^{-}$which is in disagreement with the calculated value of Bruna et al. (1980). Recently, Liu et al. (2016) investigated the low-lying 10 electronic states with spin orbit interaction of the $\mathrm{SiN}^{+}$ molecular ion by using MRCI+Q calculation.

By using a high-level ab initio MRCI $+\mathrm{Q}$ calculation, we investigate in the present work, the potential energy curves (PEC's) for 37 singlet, triplet and quintet electronic states of the $\mathrm{SiN}^{+}$molecular ion up to $140000 \mathrm{~cm}^{-1}$. The permanent dipole moment in terms of the internuclear distance $\mathrm{R}$ have been calculated for these electronic states along with the spectroscopic constants $T_{e}, R_{e}, \omega_{e}$ and $B_{e}$. The comparison of these results with those reported in the literature showed a very good agreement. Fourteen new electronic states are investigated here for the first time. 


\section{Method}

The low-lying singlet, triplet and quintet electronic states of the molecular ion $\mathrm{SiN}^{+}$have been investigated by using the Complete Active Space Self Consistent Field (CASSCF) procedure followed by a multireference configuration interaction (MRCI $+\mathrm{Q}$ with Davidson correction). The entire CASSCF configuration space was used as the reference in the MRCI calculations. The calculation has been done via the computational chemistry program MOLPRO (MOLPRO package) taking advantage of the graphical user interface GABEDIT (Allouche, 2011). The silicon atom is treated as a system of 14 electrons by using the Ahlirchs-PVDZ;c basis set for s, p, and $\mathrm{d}$ functions. The 6 electrons of the carbon species are considered using the aug-cc-pVTZ; $\mathrm{c}$ basis set for $\mathrm{s}, \mathrm{p}$ and $\mathrm{d}$ functions. Among the 20 electrons explicitly considered for $\mathrm{SiN}^{+}$molecule 6 valence electrons were explicitly treated, corresponding to 15 active orbitals. Being an heteronuclear diatomic molecule, $\mathrm{SiN}^{+}$is of $\mathrm{C}_{\infty \mathrm{v}}$ point group symmetry; however, MOLPRO software can only make use of Abelian point groups which means that $\mathrm{C}_{\infty \mathrm{v}}$ will be treated using the subgroup $\mathrm{C}_{2 \mathrm{v}}$ point group placing the molecule along the $+\mathrm{z}$-axis and keeping $\mathrm{Si}$ at the origin.

\section{Results}

\subsection{Potential Energy Curves}

The potential energy curves (PECs), in terms of the internuclear distance R, for the 36 singlet, triplet and quintet electronic states, in the representation ${ }^{2 s+1} \Lambda^{ \pm}$of the molecular ion $\mathrm{SiN}^{+}$were generated using the MRCI+Q for 101 internuclear distances. These curves are given in Figures 1-6.

The binding forces between the atoms of a molecule are the main origin of the physical properties of this molecule. The magnitude of these force are functions of the internuclear distance and they are either attractive or repulsive. The depth of a potential energy curve indicates the strength of the bond in order that the deepest potential energy curve belongs to the more stable molecule. From the drawn potential energy curves, in figures 1-6 for the molecular ion $\mathrm{SiN}^{+}$, one can notice the deep potential wells for the low triplet and singlet electronic states and shallower wells for the higher excited electronic states. While most of the potential energy curves of the quintet electronic states are shallow. From the 37 investigated electronic states 26 are bounded while the other electronic states are unbound. For these states when the electronic clouds surrounding the two atoms start to overlap, the energy of the system increases abruptly in order that one repulses each other according to Pauli principle. Moreover, some crossings and avoided crossings have been obtained between the potential energy curves in Figures 1-6. Two potential energy curves belonging to states of different symmetry they cross each other where their wavefunctions remain unperturbed and they are the adiabatic solutions of the Schrödinger equation, while the solution becomes diabatic if these wavefunctions have the same symmetry. But by linear combinations of the diabatic wavefunctions, where the variation method is used to solve for the coefficients, one can have the adiabatic solution for these states. In this case the wave functions will mix with each other where no longer cross and the crossing become avoided.

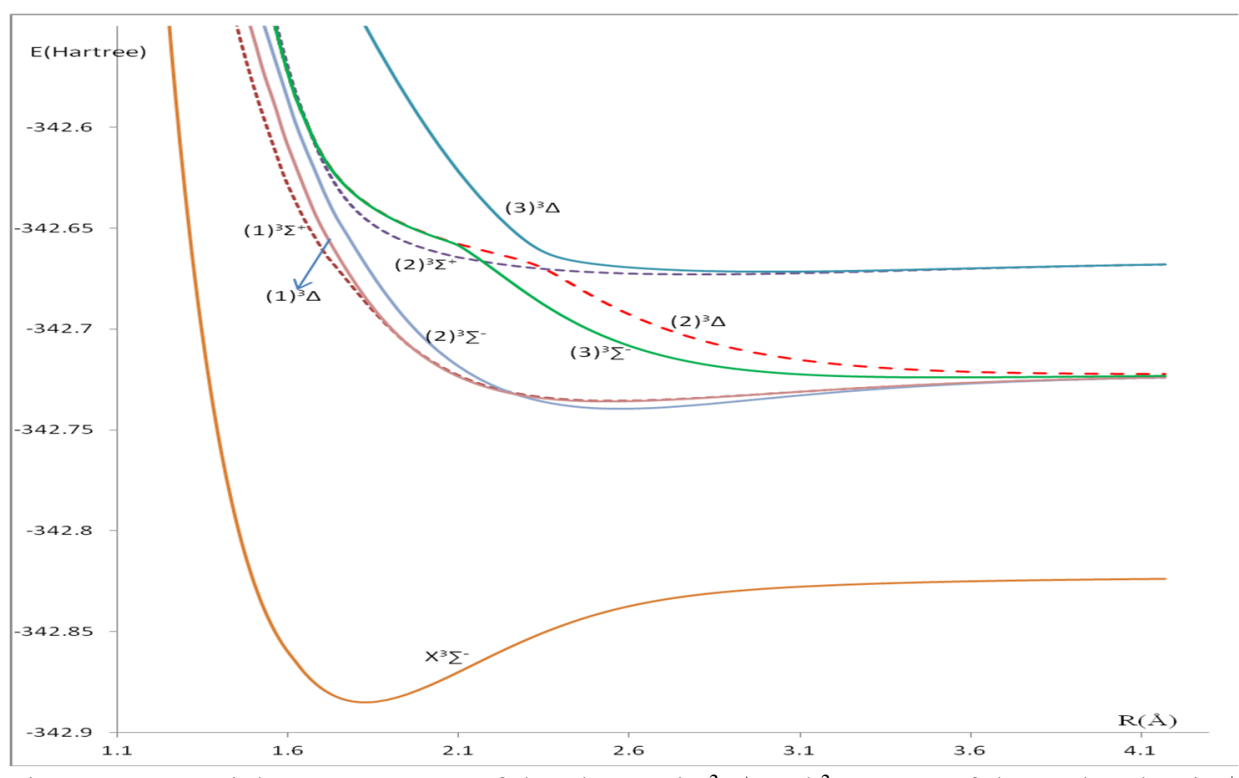

Figure 1. Potential energy curves of the electronic ${ }^{3} \Sigma^{ \pm}$and ${ }^{3} \Delta$ states of the molecule $\mathrm{SiN}^{+}$ 


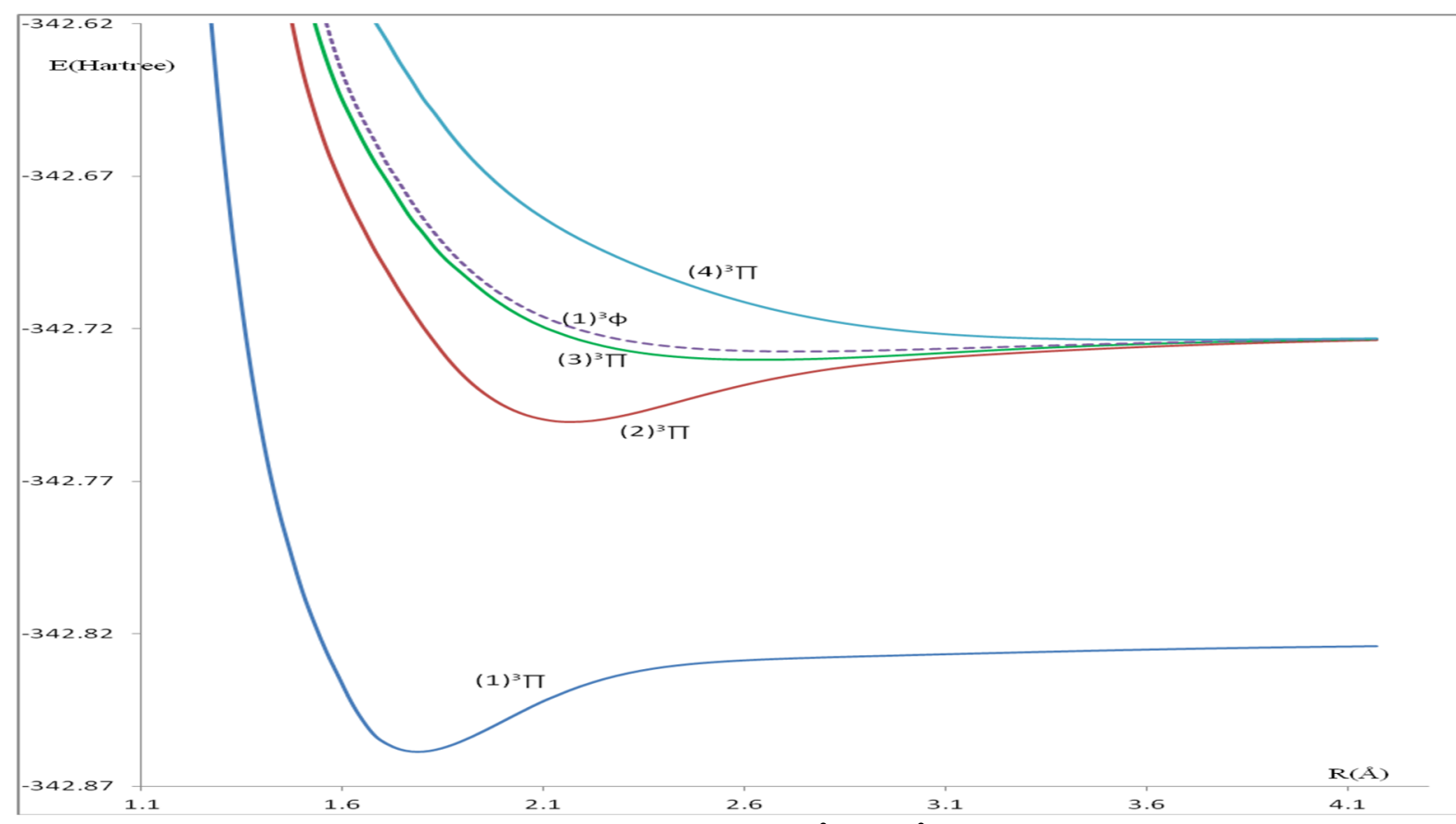

Figure 2. Potential energy curves of the electronic ${ }^{3} \Pi$ and ${ }^{3} \Phi$ states of the molecule $\mathrm{SiN}^{+}$

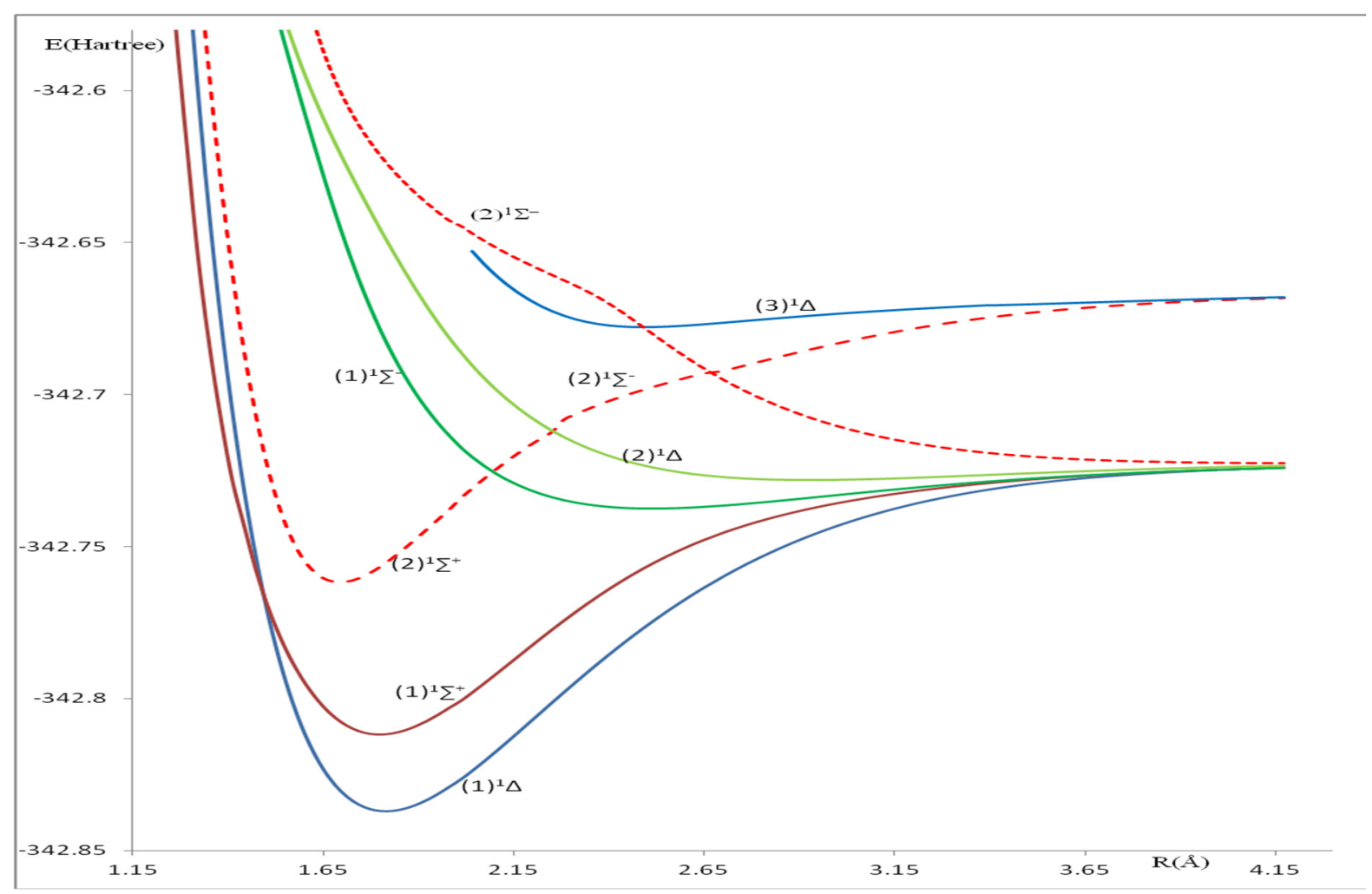

Figure 3. Potential energy curves of the electronic ${ }^{1} \Sigma^{ \pm}$and ${ }^{1} \Delta$ states of the molecule $\mathrm{SiN}^{+}$ 


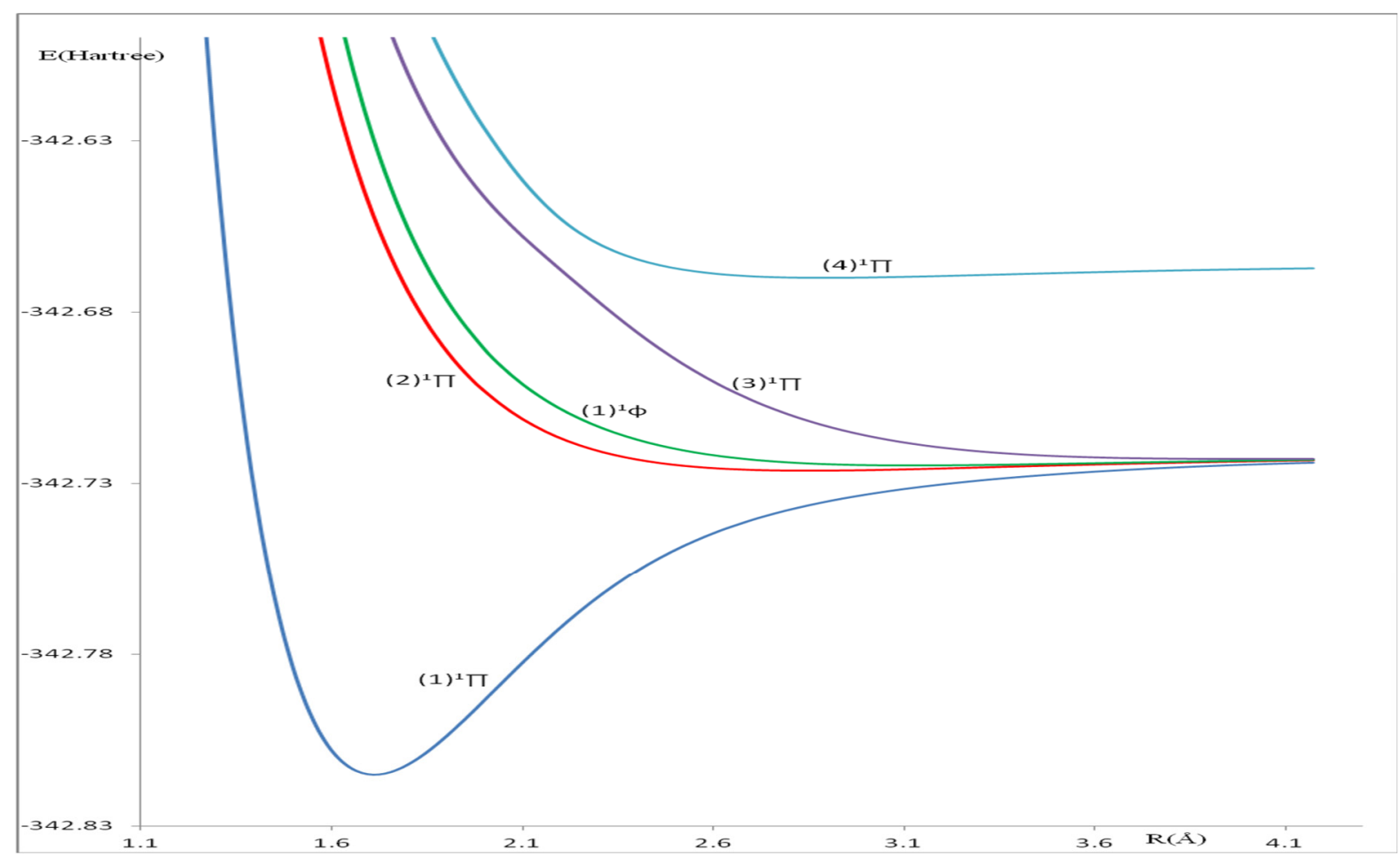

Figure 4. Potential energy curves of the electronic ${ }^{1} \Pi$ and ${ }^{1} \Phi$ states of the molecule $\mathrm{SiN}^{+}$

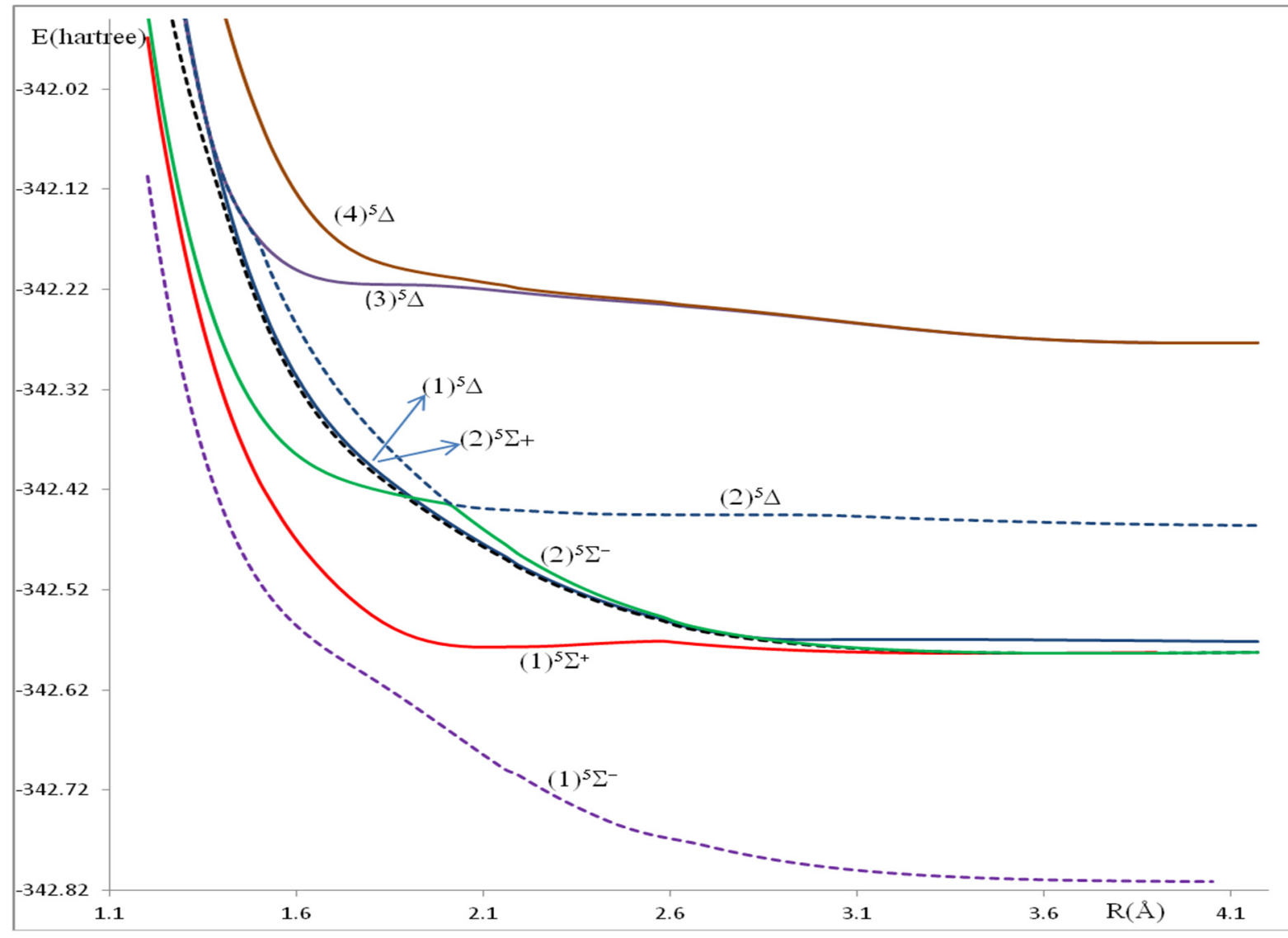

Figure 5. Potential energy curves of the electronic ${ }^{5} \Sigma^{ \pm}$and ${ }^{5} \Delta$ states of the molecule $\mathrm{SiN}^{+}$ 


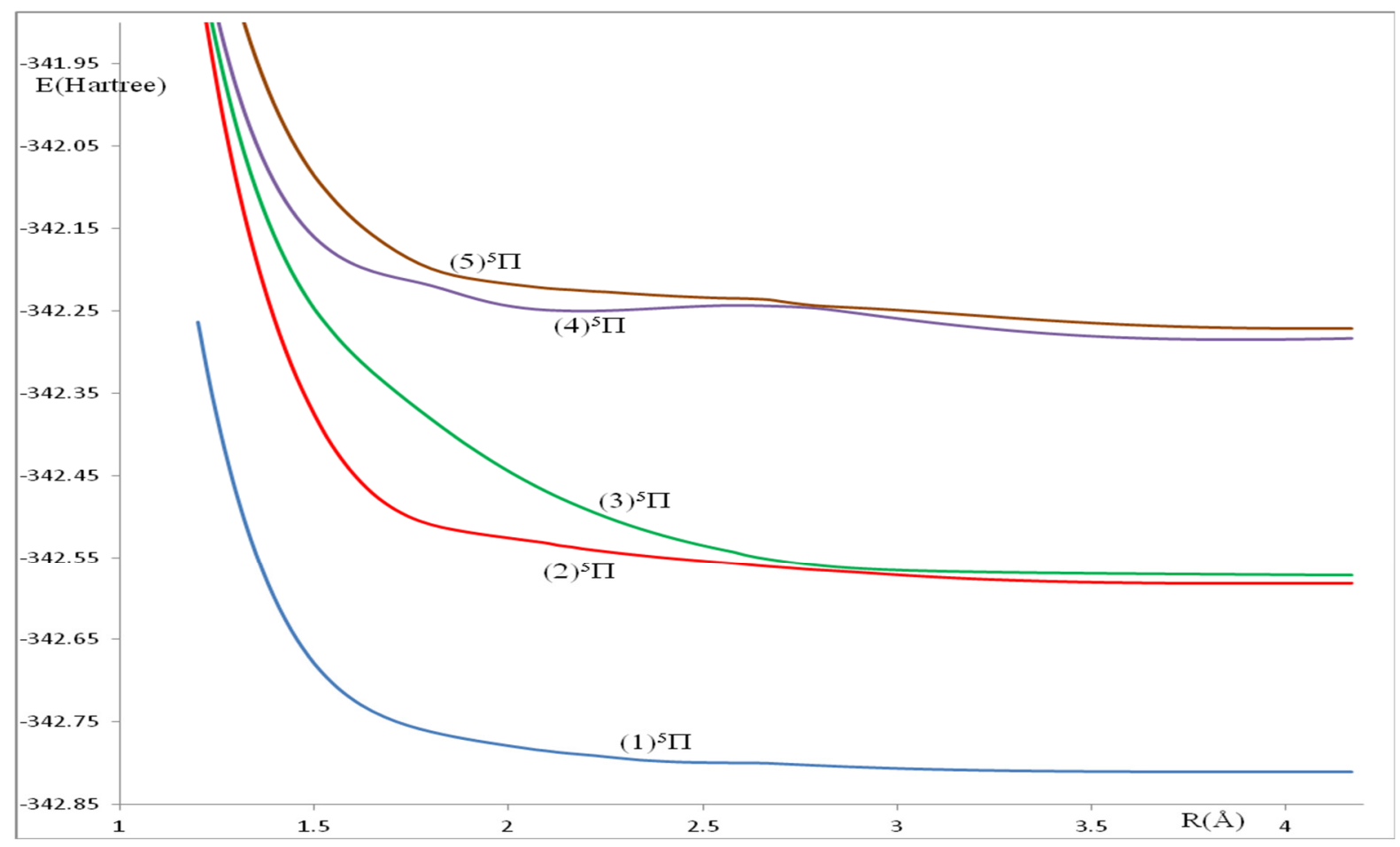

Figure 6. Potential energy curves of the electronic ${ }^{5} \Pi$ states of the molecule $\mathrm{SiN}^{+}$

\subsection{Spectroscopic Constants}

For the investigated bound electronic states of the molecular ion $\mathrm{SiN}^{+}$the transition energy with respect to the energy minimum for the ground state $T_{e}$, the equilibrium internuclear distance $R_{e}$, the harmonic frequency $\omega_{e}$ and the rotational constant $B_{e}$ have been calculated by fitting the calculated energy values of the different investigated electronic states into a polynomial in terms of $\mathrm{R}$ around the internuclear distance at equilibrium $\mathrm{R}_{\mathrm{e}}$. These values along with those given in literature are given in Table 1. The comparison of our calculated values of $T_{e}$ with those given by (Liu et al., 2016) shoes the good agreement for the investigated electronic states except for the three electronic states $(1)^{3} \Pi,(1)^{3} \Sigma^{+}$and $(1)^{1} \Sigma^{+}$where our potential energy curves are shifted up. Moreover this discrepancy can be noticed between the values given in literature (Liu et al., 2016) for the electronic states $(1)^{3} \Pi$ and $(1)^{3} \Sigma^{+}$. The comparison of our calculated values of $R_{e}$ with those given in literature shoes a very good agreement, except the 2 electronic states $(1)^{3} \sum^{+}$and $(3)^{3} \prod$ which are displaced to the right by $\sim 1 \AA$. Similar good agreement is obtained by comparing the present calculated values of $B_{e}$ with those given in literature except for the (3) $)^{3} \prod,(2)^{1} \Delta$ and (1) $)^{1} \Phi$ where our values are systematically smaller than those of (Liu et al., 2016). Concerning the investigated values of $\omega_{\mathrm{e}}$, there is a good agreement with those given in literature for $\mathrm{T}_{\mathrm{e}}<33000 \mathrm{~cm}^{-1}$, this agreement becomes less or deteriorate for some electronic states by comparison with those given by (Liu et al., 2016). The spectroscopic constants have not been calculated for the unbound electronic states and those where there is crossing or avoiding crossing near their minima of the potential energy curves $\mathrm{R}_{\mathrm{e}}$.

\subsection{Permanent Dipole Moment.}

The investigation of the dipole moment of a molecule is very useful in the study of the strength of the long range dipole-dipole forces and the understanding of the macroscopic properties of imperfect gases. From its calculation in terms of the internuclear distance, we can study the mobility of electrons through a polar gas. In the present work we place the silicon atom at the origin and the nitrogen atom along the positive direction of the internuclear axis. The investigated values of the dipole moment $\mu$ vary, in the region of calculation, between $1.44 \AA \leq R \leq 4.14 \AA$ and 3.0 a.u. $\leq \mu \leq 5.0$ a.u. for the singlet, triplet and quintet electronic states. At large internuclear distances, the dipole moment curves, of the singlet and triplet investigated electronic states, increase in absolute value in the negative region which is theoretically the correct behavior for a molecular ion. While for the quintet electronic state, one part tends in the positive region and the other part in the negative region. However, this behavior points out polarized states that dissociate into ionic fragments. An avoided crossing between the potential energy curves of two electronic states of the same symmetry is the origin of the abrupt gradient change of the DMCs. 
Table 1. Speroscopic constants of the molecular ion $\mathrm{SiN}^{+}$

\begin{tabular}{|c|c|c|c|c|}
\hline States & $T_{e}\left(\mathrm{~cm}^{-1}\right)$ & $\omega_{\mathrm{e}}\left(\mathrm{cm}^{-1}\right)$ & $\mathrm{B}_{\mathrm{e}}\left(\mathrm{cm}^{-1}\right)$ & $\mathrm{R}_{\mathrm{e}}(\AA)$ \\
\hline \multirow{3}{*}{$(1)^{3} \Sigma^{-}$} & \multirow{3}{*}{0.0} & 749.6 & 0.5427 & 1.823 \\
\hline & & $777.6^{\mathrm{b}}$ & $0.5628^{\mathrm{b}}$ & $1.7972^{\mathrm{b}}$ \\
\hline & & $717^{\mathrm{c}}$ & & $1.81^{\mathrm{c}}$ \\
\hline \multirow{4}{*}{$(1)^{3} \Pi$} & 5734.2 & 852.8 & 0.5667 & 1.784 \\
\hline & $723.4^{\mathrm{b}}$ & $922.3^{\mathrm{b}}$ & $0.6384^{b}$ & $1.6825^{\mathrm{b}}$ \\
\hline & $1693.8^{c}$ & $856^{c}$ & & \\
\hline & 10512.4 & 775.6 & 0.5487 & 1.813 \\
\hline \multirow[t]{2}{*}{$(1)^{1} \Delta$} & $9570.9^{b}$ & $793.2^{b}$ & $0.5628^{b}$ & $1.7920^{\mathrm{b}}$ \\
\hline & $11453.3^{c}$ & & & $1.81^{\mathrm{c}}$ \\
\hline \multirow{3}{*}{$(1)^{1} \Pi$} & 15352.6 & 855.86 & \multirow{2}{*}{0.6150} & 1.713 \\
\hline & $16082.16^{\mathrm{c}}$ & $915^{c}$ & & $1.69^{\mathrm{c}}$ \\
\hline & 16090.0 & 733.6 & 0.5582 & 1.798 \\
\hline \multirow[t]{2}{*}{$(1)^{1} \Sigma^{+}$} & $7693.8^{b}$ & & & \\
\hline & $9840.19^{c}$ & $1464^{\mathrm{c}}$ & & $1.57^{\mathrm{c}}$ \\
\hline$(2)^{1} \sum^{+}$ & 27067.5 & 985.7 & 0.6300 & 1.692 \\
\hline \multirow{2}{*}{$(2)^{3} \Pi$} & 29489.7 & 507.26 & 0.3832 & 2.169 \\
\hline & $28330.7^{b}$ & $700.25^{b}$ & $0.5400^{b}$ & $1.926^{\mathrm{b}}$ \\
\hline \multirow{2}{*}{$(2)^{3} \Sigma^{-}$} & 31930.1 & 282.9 & 0.2717 & 2.575 \\
\hline & $34243.9^{b}$ & $326.8^{\mathrm{b}}$ & $0.2928^{b}$ & $2.4835^{b}$ \\
\hline \multirow{2}{*}{$(1)^{1} \Sigma^{-}$} & 32405.3 & 239.3 & 0.2865 & 2.511 \\
\hline & $34033.5^{b}$ & $320.9^{b}$ & $0.3256^{b}$ & $2.3560^{b}$ \\
\hline \multirow{2}{*}{$(1)^{3} \Delta$} & 32722.1 & 226.8 & 0.2803 & 2.537 \\
\hline & $34541.1^{b}$ & $308.6^{\mathrm{b}}$ & $0.3200^{b}$ & $2.36^{\mathrm{b}}$ \\
\hline \multirow{2}{*}{$(1)^{3} \Sigma^{+}$} & 32822.7 & 222.2 & 0.2774 & 2.551 \\
\hline & $22770.2^{b}$ & $1186.3^{b}$ & & $1.5385^{b}$ \\
\hline \multirow{2}{*}{$(3)^{3} \Pi$} & 34011.5 & 165.9 & 0.2592 & 2.639 \\
\hline & $30858.8^{b}$ & $779.6^{\mathrm{b}}$ & $0.5189^{b}$ & $1.8745^{\mathrm{b}}$ \\
\hline \multirow{2}{*}{$(1)^{3} \Phi$} & 34597.4 & 130.9 & 0.2426 & 2.727 \\
\hline & $36046.1^{b}$ & $269.4^{\mathrm{b}}$ & $0.3976^{b}$ & $2.1325^{b}$ \\
\hline \multirow{2}{*}{$(2)^{1} \Delta$} & 34439.7 & 136.3 & 0.2062 & 2.933 \\
\hline & $37111.5^{b}$ & $210.1^{b}$ & $0.4947^{b}$ & $2.6545^{b}$ \\
\hline$(2)^{1} \Pi$ & 34856.6 & 62.6 & 0.2099 & 2.8494 \\
\hline \multirow{2}{*}{$(1)^{1} \Phi$} & 35159.7 & 127.5 & 0.1828 & 3.125 \\
\hline & $38266.0 \mathrm{~b}$ & $144.1^{\mathrm{b}}$ & $0.2554^{b}$ & $2.65^{b}$ \\
\hline \multirow{2}{*}{$(3)^{3} \Sigma^{-}$} & 35372.7 & 82.4 & 0.1447 & 3.5273 \\
\hline & $38836.7^{b}$ & $100.4^{\mathrm{b}}$ & $0.1732^{b}$ & $3.2305^{b}$ \\
\hline \multirow{2}{*}{$(4)^{3} \Pi$} & 35446.1 & 69.9 & 0.1366 & 3.6300 \\
\hline & $38282.9^{b}$ & $860.9^{b}$ & $0.510^{\mathrm{b}}$ & $1.8715^{b}$ \\
\hline$(3)^{1} \Delta$ & 35480.3 & 769.8 & 0.2793 & 2.542 \\
\hline \multirow{2}{*}{$(3)^{1} \Pi$} & 35589.9 & 171.8 & & 3.973 \\
\hline & $39240.5^{b}$ & $173.3^{b}$ & & $3.83^{b}$ \\
\hline$(3)^{1} \Sigma^{+}$ & 45480.3 & 306.2 & 0.2906 & 2.490 \\
\hline$(2)^{3} \Sigma^{+}$ & 46571.0 & 114.0 & 0.2245 & 2.806 \\
\hline$(3)^{3} \Delta$ & 46873.01 & 516.2 & 0.1709 & 2.994 \\
\hline$(4)^{1} \Pi$ & 47188.2 & 119.4 & 0.2164 & 2.891 \\
\hline$(1)^{5} \Sigma^{+}$ & 67661.3 & 397.5 & 0.4018 & 2.114 \\
\hline$(4)^{5} \Pi$ & 139354.9 & 441.7 & 0.3759 & 2.190 \\
\hline
\end{tabular}


For the considered electronic states of the molecular ion $\mathrm{SiN}^{+}$a reversed polarity of the corresponding atoms takes place at each avoided crossing region as indicated from the sharp change in the slopes of the DMCs of the states at which the dipole moment changes its gradient sign. There is no comparison of these investigated values of the dipole moments since they are calculated here for the first time. The variation of the dipole moment curves, in terms of the internuclear distance $\mathrm{R}$, for singlet, triplet and quintet electronic states on $\mathrm{SiN}^{+}$cation are given in Figures 7-12.

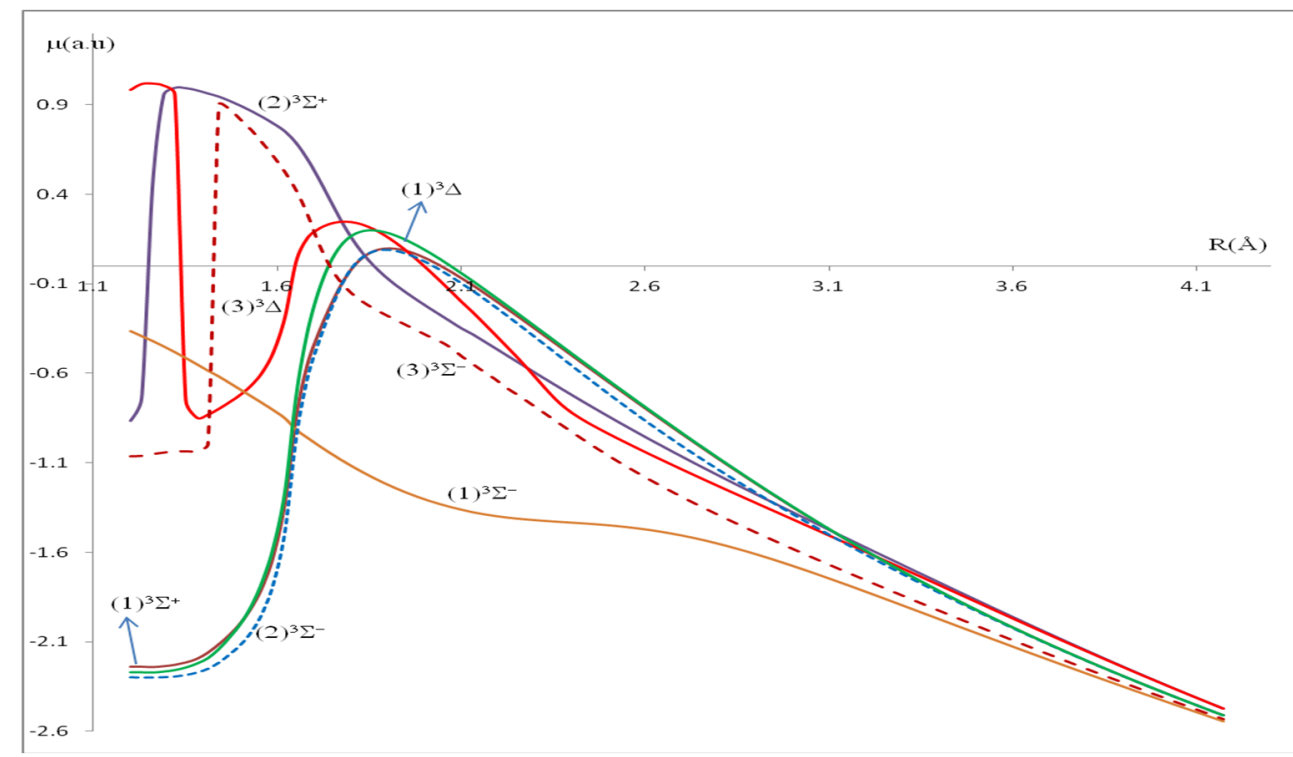

Figure 7. Dipole moment curves of ${ }^{3} \Sigma^{ \pm}$and ${ }^{3} \Delta$ electronic states of the molecular ion $\mathrm{SiN}^{+}$

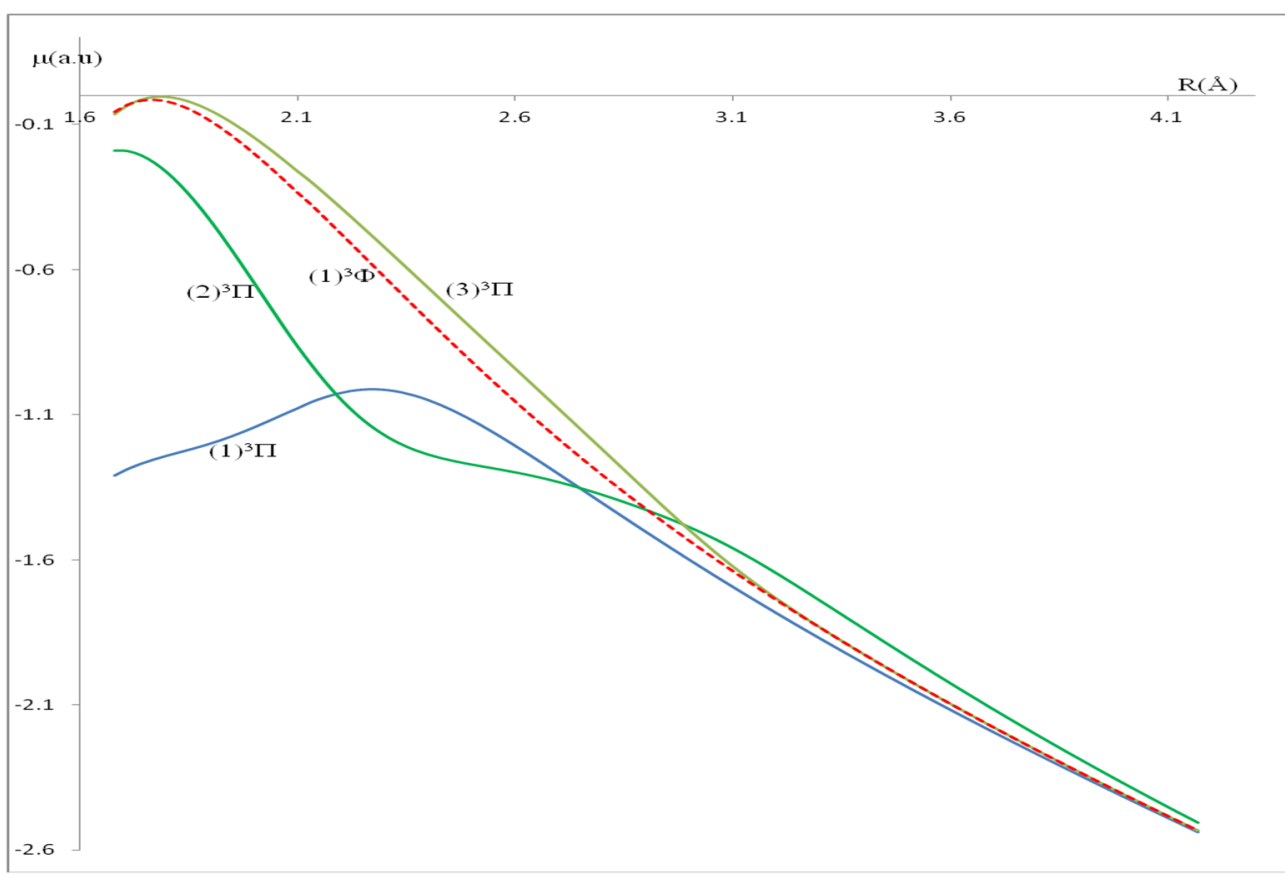

Figure 8. Dipole moment curves of ${ }^{3} \Pi$ and ${ }^{3} \Phi$ electronic states of the molecular ion $\mathrm{SiN}^{+}$ 


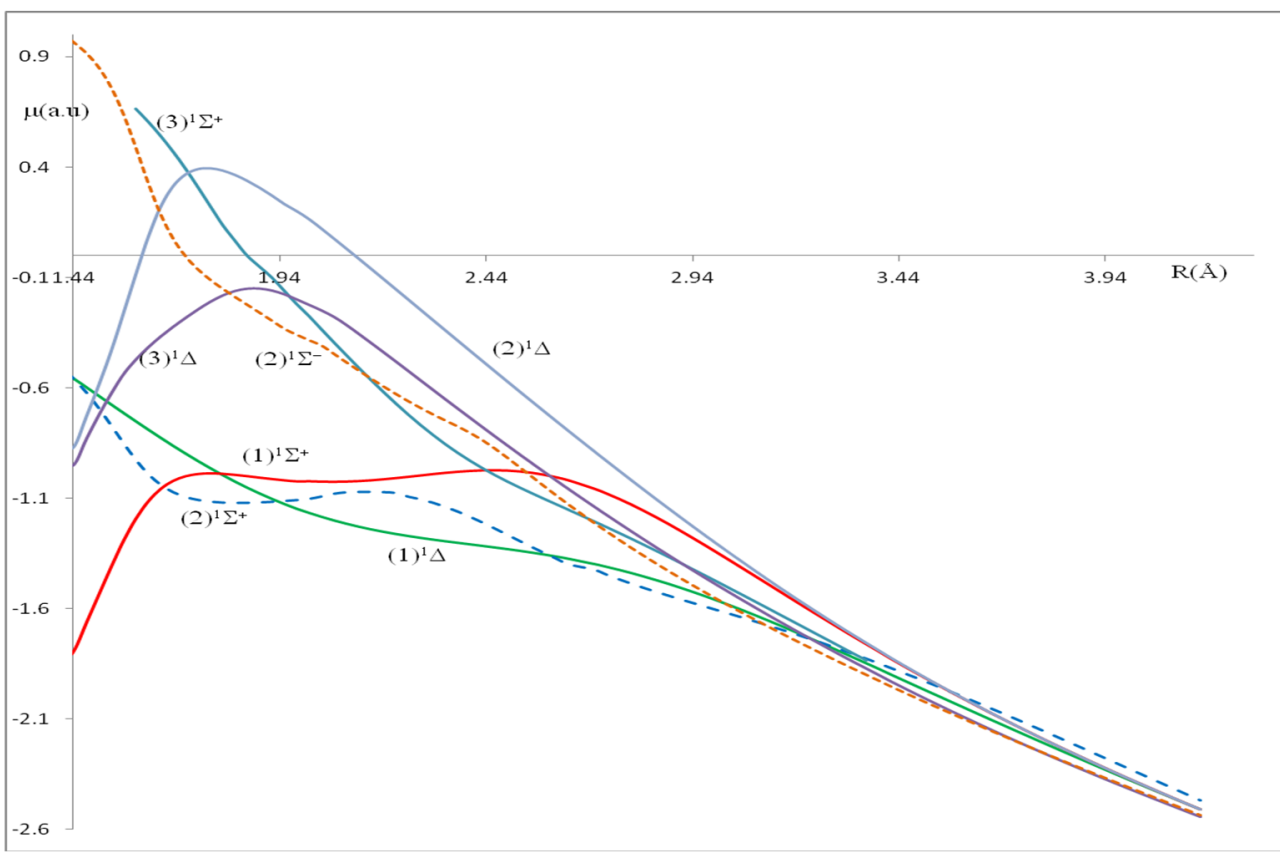

Figure 9. Dipole moment curves of ${ }^{1} \Sigma^{ \pm}$and ${ }^{1} \Delta$ electronic states of the molecular ion $\mathrm{SiN}^{+}$

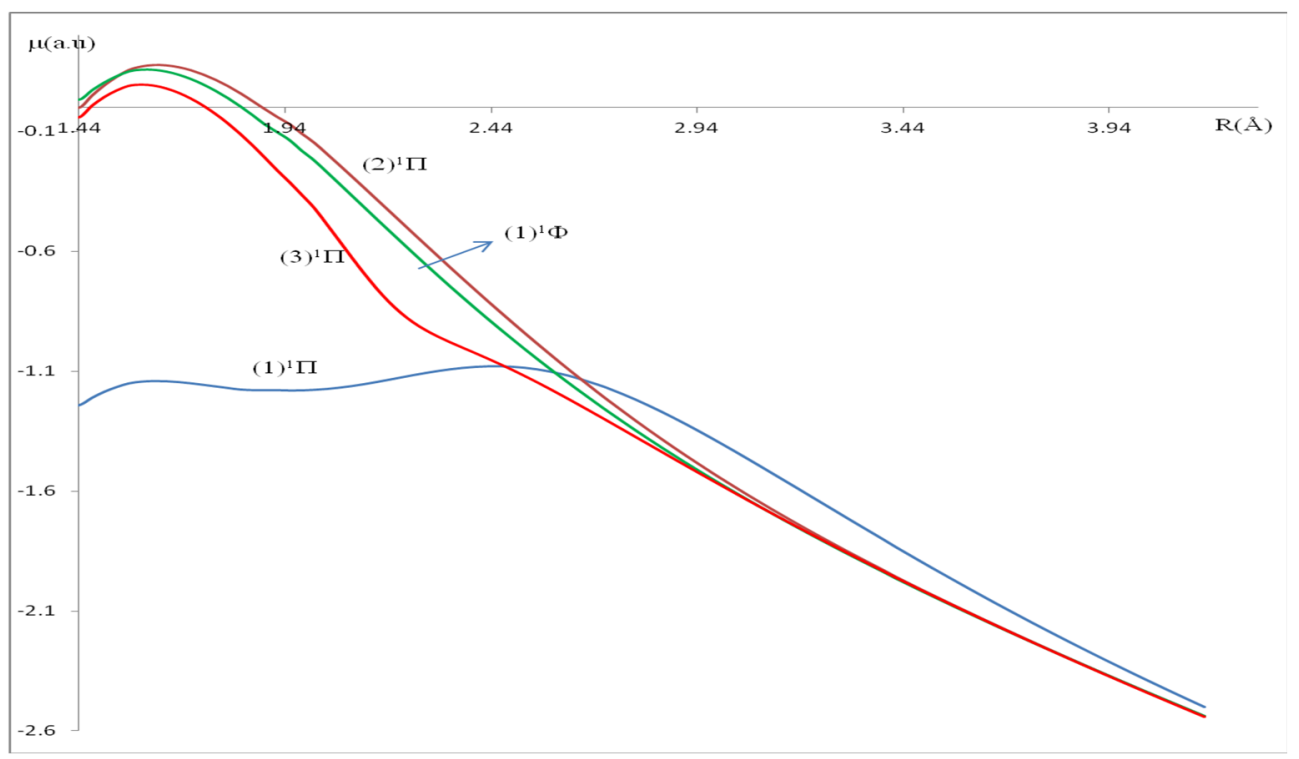

Figure 10. Dipole moment curves of ${ }^{1} \Pi$ and ${ }^{1} \Phi$ electronic states of the molecular ion $\mathrm{SiN}^{+}$ 


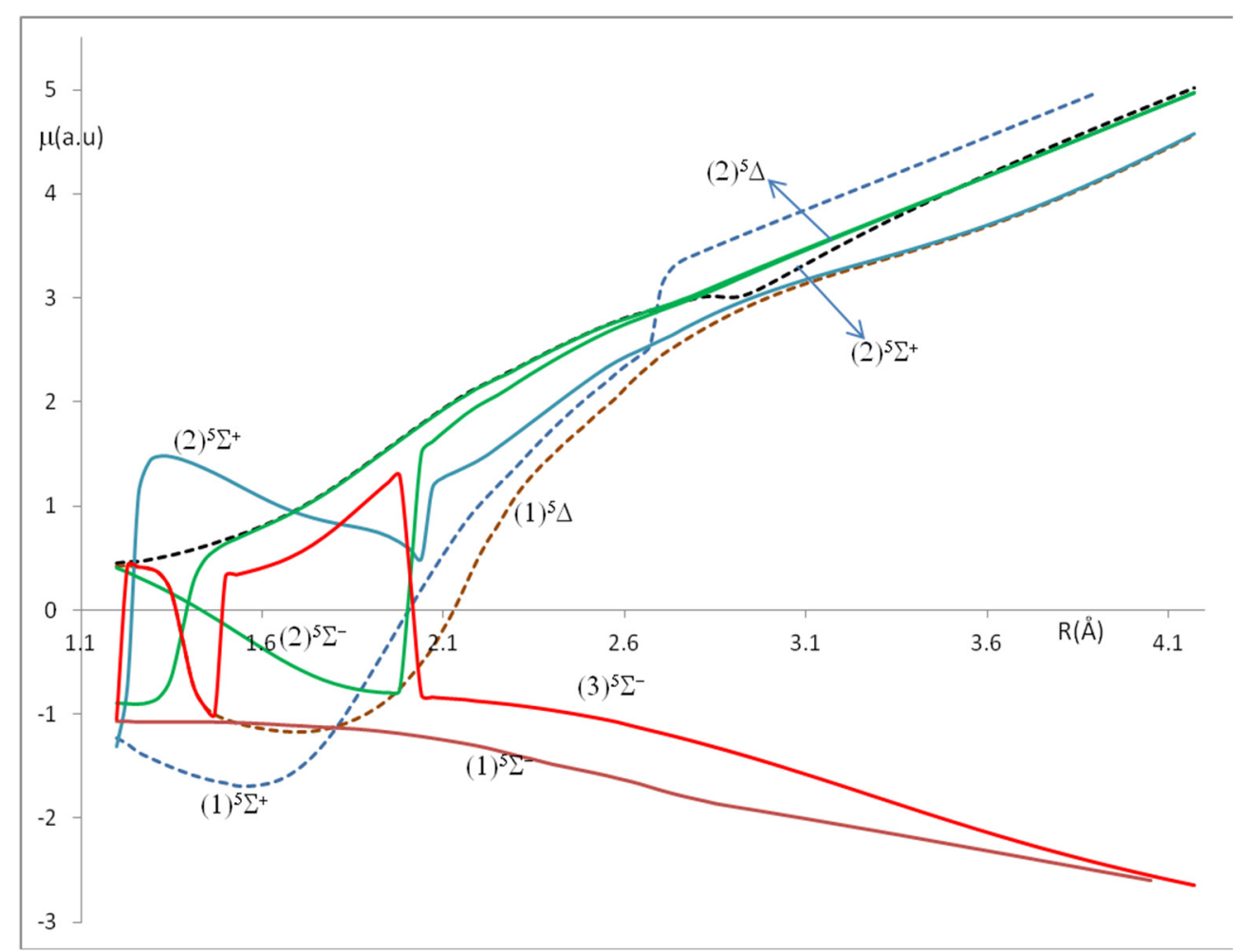

Figure 11. Dipole moment curves of ${ }^{5} \Sigma^{ \pm}$and ${ }^{5} \Delta$ electronic states of the molecular ion $\mathrm{SiN}^{+}$

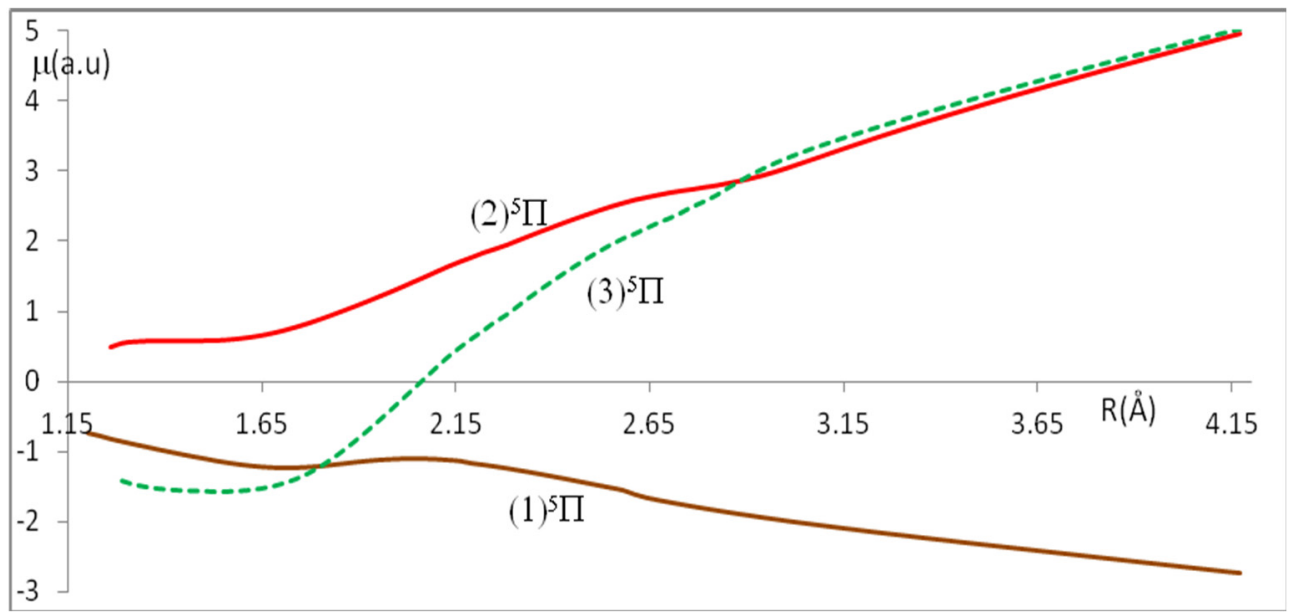

Figure 12. Dipole moment curves of ${ }^{5} \Pi$ electronic states of the molecular ion $\mathrm{SiN}^{+}$

\section{Conclusion}

The static dipole moment and the potential energy curves of 37 singlet, triplet and quintet electronic states of the molecule ion $\mathrm{SiN}^{+}$have been investigated by using a high precision level ab initio $\mathrm{MRCI}+\mathrm{Q}$ calculation with Davidson correction. The spectroscopic constants $T_{e}, R_{e}, \omega_{e}$ and $B_{e}$ have been calculated for the bounded states up to to $140000 \mathrm{~cm}^{-1}$. The comparison of these calculated values with those given in literature shows an overall good agreement. At large internuclear distances, the dipole moment curves, of the singlet and triplet investigated electronic states, increase in absolute value in the negative region while for the quintet electronic state, one part tends in the positive region and the other part in the negative one. All these dipole moment curves along with nine electronic states have been studied in the present work for the first time. These new results may stimulate more investigation of experimental work on this molecular ion $\mathrm{SiN}^{+}$. 


\section{Acknowledgements}

The authors would like to thank the Lebanese National Council for Scientific Research for the financial support of the present work by the grant program for scientific research 2013-2015.

\section{References}

Allouche, A. R. (2011). Gabedit: A graphical user interface for computational chemistry software. J. Comp. Chem. 32, 174-182. http://dx.doi.org/10.1002/jcc. 21600

Bredohl, H., Dubois, I., Houbrechts, Y., \& Singh, M. (1976). The emission spectrum of SiN. Can. J. Phys. 54, 680-688. http://dx.doi.org/10.1139/p76-76

Bruna, P. J., Peyerimhoff, S. D., \& Buenker, R. J. (1980). Theoretical prediction of the potential curves for the lowest - lying states of the isovalent diatomics $\mathrm{CN}+, \mathrm{Si} 2, \mathrm{SiC}, \mathrm{CP}+$, and $\mathrm{SiN}+$ using the ab initio MRD - CI method. J. Chem. Phys., 72, 5437. http://dx.doi.org/10.1063/1.439012

Cai, Z. L., \& François, J. P. (1999). Ab initio study of the lowest 3Sigma-, 3Pi and 1Sigma+ states of the SiN+ cation. Chem. Phys. Lett., 300, 69-79. http://dx.doi.org/10.1016/S0009-2614(98)01329-3

Elhanine, M., Hanoune, B., Guelachvili, G., \& Amiot, C. (1992). The infrared emission spectrum of SiN between 2.2 and $4.4 \mu \mathrm{m}$. J. Phys. France, 24, 931-938. http://dx.doi.org/10.1051/jp2:1992176

Foster, S. C. (1984). The B2 $+\rightarrow$ A2Пi system of silicon nitride. SiN. J. Mol. Spectrosc, 106, 369-375. http://dx.doi.org/10.1016/0022-2852(84)90167-X

Foster, S. C., Lubic, K. G., \& Amano, T. (1985). The $2-0$ band of the A2Пi $\leftarrow$ X $2 \Sigma+$ system of SiN near $3.3 \mu \mathrm{m} . J$. Chem. Phys., 82(2) 709-713. http://dx.doi.org/10.1063/1.448548

Foster, S. C. (1989). The vibronic structure of the SiN Radical. J. Mol. Spectrosc, 137, 430-431. http://dx.doi.org/ 10.1016/0022-2852(89)90185-9

Goldberg, N., Iraqi, M., Schwarz, H., Boldyrev, A., \& Simons, J. (1994). A combined experimental and theoretical study of the neutral, cationic, and anionic Si3N cluster molecule. J. Chem. Phys., 101, 2871. http://dx.doi.org/ $10.1063 / 1.467601$

Ito, H., Suzuki, K., Kondow, T., \& Kuchitsu, K. (1993). An analysis of the B2 $2+-X 2 \Sigma+$ emission of SiN. Dependence of the electronic transition moment on the SiN internuclear distance. Chem. Phys. Lett., 208(3-4), 328-334. http://dx.doi.org/10.1016/0009-2614(93)89084-U

Katz, R. N. (1980). High-temperature structural ceramics. Science, 208, 841-847. http://dx.doi.org/10.1126/ Science.208.4446.841

Linton, C. (1975). Rotational analysis of some Mulliken D2П-A2П bands of the SiN molecule. J. Mol. Spectrosc, 55, 108-119. http://dx.doi.org/10.1016/00222852(75)90256-8

Liu, Y. F., Zhai, H., \& Liu, Y. L. (2015). Extensive ab initio calculation on low-lying excited states of SiN + cation including spin-orbit coupling. Eur. Phys. J. D, 69, 59. http://dx.doi.org/10.1140/epjd/e2015-50584-y

Naulin, C., Costes, M., Moudden, Z., Ghanem, N., \& Dorthe, G. (1993). The dissociation energy of the SiN radical determined from a crossed molecular beam study of the $\mathrm{Si}+\mathrm{N} 2 \mathrm{O} \rightarrow \mathrm{SiN}+\mathrm{NO}$ reaction. Chem. Phys. Lett., 202(5), 452-458. http://dx.doi.org/10.1016/0009-2614(93)90069D

Molpro Quantum Chemistry Software. (2008). MOLPRO is a package of ab initio programs. Retrieved from http://www.molpro.net/info/users

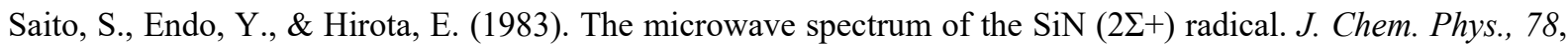
6447. http://dx.doi.org/10.1063/1.444682

Yamada, C., \& Hirota, E. (1985). The A2Пi $-\mathrm{X} 2 \Sigma+$ transition of the SiN radical by infrared diode laser spectroscopy. J. Chem. Phys., 82, 2547. http://dx.doi.org/10.1063/1.448304

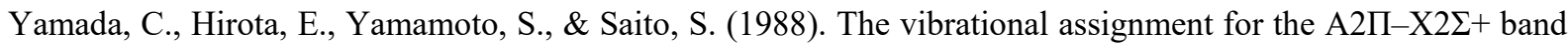
system of the SiN radical: The $0-0$ bands of $29 \mathrm{SiN}$ and 30SiN. J. Chem. Phys., 88, 46. http://dx.doi.org/10.1063/1.454627

\section{Copyrights}

Copyright for this article is retained by the author(s), with first publication rights granted to the journal.

This is an open-access article distributed under the terms and conditions of the Creative Commons Attribution license (http://creativecommons.org/licenses/by/3.0/). 